Ann. Biol. anim. Bioch. Biophys., 1979, 19 (6), 1817-1826.

\title{
Etude cytophotométrique de l'ADN et des nucléoprotéines des spermatozoïdes du bélier : influence du séjour dans les voies génitales de la brebis
}

\author{
par J. C. NICOLLE, C. ESNAULT, M. COUROT
}

Station de Physiologie de la Reproduction, I. N. R. A. Nouzilly, 37380 Monnaie.

Summary. A cytophotometric study of ram spermatozoon DNA and nucleoproteins.

Ejaculated ram spermatozoa were microspectrophotometrically observed as such (controls) or after incubation in the female genital tract ( 2 to $24 \mathrm{hrs}$ ). The nuclear DNA content, measured with UV light, did not significantly differ whether the spermatozoa were incubated in utero or not. However, quantitative measurements of various components, carried out with visible light on specifically stained cells, indicated that the sperm staining abilities were different. The Feulgen DNA increased in 60 p. 100 of the sperm samples incubated in ufero. After the longest incubation periods, this was especially evident in 7 out of 8 ewes and 10 out of 10 ewes after 22 and 24 hrs, respectively. In these cases, the mean values for Feulgen DNA extinctions were increased by 21 and 29 p. 100 . Most of the in utero-incubated samples $(19 / 21)$ had the highest extinction values when stained with Fast Green for arginine-rich basic proteins. The nuclear area of in utero-incubated sperm was increased as compared to the non-incubated one. This increase was especially evident after an 18 to 24 hrs incubation. Differences in staining ability would be due to modifications in chromatin structure. The significance of these changes in relation to the preparation of spermatozoa for fertilization has been discussed. The appearance of abnormalities on some spermatozoa, seen after staining with Fast-Green, might indicate a deleterious effect of the uterine environment on already altered spermatozoa.

\section{Introduction.}

La structure condensée de la chromatine des spermatozoïdes de bélier résulte de la formation, pendant les dernières phases de la spermatogenèse, d'un complexe entre l'ADN et la protéine spécifique du spermatozoïde (BNSP) riche en cystéine et en arginine (Loir, 1972a ; Monfoort et al., 1973). La mise en place progressive de ponts disulfures, permettant une liaison plus étroite des protéines entre elles, accentue ce phénomène ; elle se poursuit pendant la maturation épididymaire (Marushige et Marushige, 1975). Cette condensation s'accompagne d'une diminution de colorabilité de l'ADN par le Feulgen sans que la teneur en ADN des spermatozoïdes, mesurée en UV ne diminue (Esnault, 1965 ; Gledhill ef al., 1966 ; Esnault et Nicolle, 1976). 
Nous avons cherché à connaître la manière dont évolue la chromatine des spermatozoïdes après l'éjaculation, lorsqu'ils séjournent dans l'appareil génital femelle. Nous rapportons dans le présent travail, les résultats d'une étude microspectrophotométrique des spermatozoïdes de bélier après un séjour prolongé dans les voies génitales de la brebis.

\section{Matériel et méthodes.}

Cette étude a été réalisée au cours de la saison sexuelle pendant trois années consécutives. 98 brebis de race lle-de-France régulièrement cyclées (observation préalable pendant 2 cycles) ont été saillies par des béliers de même race, dans les premières heures $(1$ à $6 \mathrm{~h}$ ) des chaleurs naturelles. 8 brebis ont été sacrifiées toutes les $2 \mathrm{~h}$ entre $2 \mathrm{~h}$ à $24 \mathrm{~h}$ après la saillie (10 q à 24 h), en vue de récupérer les spermatozoïdes présents dans l'utérus.

1. Récolte et préparation des échantillons.

a) Récolte des spermatozoïdes « utérins». - Les cornes utérines, attachées au cervix ont éfé prélevées dès l'abattage des brebis. Les spermatozoïdes ont été recueillis par perfusion séparée de chacune des cornes utérines avec $0,5 \mathrm{ml}$ de tampon Ringer formolé, à partir de l'oviducte. L'addition de formol au milieu de Ringer $(1 \mathrm{ml} / \mathrm{l})$ assurait une préfixation des spermatozoïdes. Ceux-ci, contenus dans le perfusat récupéré à l'extrémité " cervicale » du corps utérin, ont été déposés sur lames par cytocentrifugation (200 g pendant $10 \mathrm{~min}$ ). Cette technique a permis de concentrer les cellules direciement sur une lame histologique, en une plage circulaire de $5 \mathrm{~mm}$ de diamètre à partir de petits volumes $(0,2 \mathrm{ml})$ de suspensions faiblement concentrées.

b) Récolte des spermatozoïdes éjaculés. - Ce sont les spermatozoïdes témoins ; ils n'ont eu aucun contact avec les sécrétions génitales femelles car ils ont été prélevés, directement sur le bélier, aussitôł la saillie : 1 goutte de sperme était mise en suspension dans 2 à $3 \mathrm{ml}$ de tampon Ringer formolé et les étalements de spermatozoïdes ont été préparés comme précédemment. Un contrôle de la qualité du sperme (appréciation de vitalité sous microscope) a été effectué sur une suspension dans le milieu de Ringer sans formol.

A titre de contrôle de l'effet propre éventuel de la température des voies génitales femelles, des spermatozoïdes de 18 éjaculats ont été incubés in vitro à $38^{\circ} \mathrm{C}$ pendant $1,2,3,17,20$ et $24 \mathrm{~h}$.

c) Préparation des échantillons. - Les préparations de spermatozoïdes éjaculés et utérins réalisées sur lames de quartz et de verre ont été fixées pendant $15 \mathrm{~min}$ aux vapeurs de formol neutre (les préparations destinées à être colorées par le Fast Green subissaient une fixation supplémentaire de $45 \mathrm{~min}$ dans le formol neutre à 10 p. 100). Les lames de quartz ont été déshydratées (éthanol 950, $2 \times 10 \mathrm{~min}$ - éthanol absolu $2 \times 10 \mathrm{~min}$ ) et montées à la glycérine après séchage à l'air, pour permettre des mesures direciement en lumière UV. Les préparations sur lames de verre ont été séchées à l'air et conservées au froid $\left(+4^{\circ} \mathrm{C}\right)$ à l'abri de la lumière pour être colorées ultérieurement. 


\section{Colorations - Mesures.}

Les mesures d'absorption ont été effectuées à l'aide du microspectrophotomètre UMSP I Zeiss par balayage fotal du noyau de 30 spermatozoïdes par échantillon ef intégration des densités optiques suivanf la méthode de Caspersson (1936). Les résultats sont exprimés en unités arbitraires proportionnelles à la teneur d'un noyau de spermatozoïde en matériel recherché :

- en lumière ultraviolette $(\lambda=265 \mathrm{~nm})$ sur lames de quartz pour la détermination de la teneur en ADN, appelée « ADN-UV » (avec correction pour la perte de lumière non spécifique mesurée à $\lambda=315 \mathrm{~nm}$ ) (Esnault 1973) ;

- en lumière visible sur lame de verre :

- à $\lambda=560 \mathrm{~nm}$, après coloration selon la technique de Feulgen (lavage à l'eau $1 \mathrm{~h}$ pour éliminer l'excès de fixateur, hydrolyse $\mathrm{HCl} / \mathrm{N}-60^{\circ}-10 \mathrm{~min}$, réactif de Schiff $1 \mathrm{~h}$ ) pour la détermination du matériel dit «ADN-Feulgen » (Esnault et Nicolle, 1976) ;

- à $\lambda=640 \mathrm{~nm}$, après coloration au Fast-Green alcalin ( $\mathrm{pH} 8,2$ ) pour l'évaluation de la teneur en protéines basiques (Alfert et Geschwind, 1953). L'extraction préalable des acides nucléiques était réalisée à $90^{\circ} \mathrm{C}$ par du TCA 8 p. 100 (Esnault et Nicolle, 1976).

Dans le cas présent, seules les protéines basiques riches en arginine sont mises en évidence, la lysine ayant disparu au début de l'allongement des spermatides (Loir, 1972b).

- à $\lambda=590 \mathrm{~nm}$, après réaction de Barrnett et Seligman (1952) modifiée par l'emploi du Fast-Black $K$ après couplage au DDD (Bahr et Moberger, 1958), pour la mise en évidence des groupements disulfures réduits par le thioglycérol. Les groupements SH libres étaient préalablement bloqués par l'acide iodoacétique.

La mesure de la surface du noyau des spermatozoïdes est déterminée automafiquement par le microspectrophotomètre, lors du balayage total, par les mesures d'absorption en ne prenant en compte que celles dont la valeur diffère de celle du « fond » environnant.

\section{Expression des résultats.}

- La teneur en matériel recherché est exprimée par la valeur moyenne d'extinction ( \pm erreur standard) déterminée :

1) Sur 30 spermatozoïdes éjaculés pour chaque bélier (moyenne extinction spermatozoïdes éjaculés) ;

2) Sur 30 spermatozoïdes récupérés dans l'utérus de chaque brebis (moyenne extinction spermatozoïdes utérins).

Parmi ceux-ci, il semble exister une variabilité dans les niveaux de coloration (que ce soit après Feulgen, Fast-Green ou réaction de Barrnett et Seligman). En effet, dans certains échantillons incubés in utero montrant une augmentation de coloration, il apparaît qu'une proportion sensible de spermatozoïdes ne présente pas de modification dans leur extinction par rapport aux spermatozoïdes témoins. Malheureuse- 
ment, il ne nous a pas été possible techniquement d'évaluer cette proportion par la mesure microspectrophotométrique d'un nombre assez important de spermatozoïdes (au minimum 200) ni par une numération au microscope, car dans ce cas, les différences d'intensité de coloration ne sont pas suffisamment importantes pour être appréciées par l'œil de l'observateur.

Le taux de modification du contenu des spermatozoïdes est calculé pour chaque éjaculat par le rapport :

$$
\frac{\overline{\mathrm{m}} \text {. extinction spz. Ut }-\overline{\mathrm{m}} \text {. extinction spz. Ejac }}{\overline{\mathrm{m}} \text {. extinction spz. Ejac. }} \times 100
$$

La plupart des spermatozoïdes incubés à $38^{\circ} \mathrm{C}$ dans du tampon Ringer (contrôle de l'effet de la température) révèlent une diminution de leur colorabilité au réactif de Feulgen (20 à 50 p. 100 selon les échantillons, sans liaison apparente avec la durée d'incubation) ; c'est pourquoi seuls, les échantillons de spermatozoïdes dont la colorabilité au Feulgen augmente significativement après séjour dans les voies génifales femelles seront considérés comme ayant réagi au milieu utérin.

- Les taux de modifications de surface des noyaux sont exprimés par la moyenne des pourcentages de changement de taille des spermatozoïdes « utérins » par rapport aux spermatozoïdes éjaculés.

- Lorsque le nombre de femelles est réduit, les résultats sont exprimés par la moyenne des valeurs individuelles d'extinction (moyenne de 30 spermatozoïdes par échantillon) et par leur erreur-standard. La variation du contenu en matériel recherché est alors exprimée en pourcentage (extinction des spermatozoïdes utérins/extinction des spermatozoïdes éjaculés) sans signification statistique de cette différence (tabl. 1, 3, 4).

\section{Résultats.}

a) Evolution comparée de l' «ADN-UV » et de l' "ADN-Feulgen ». - Les valeurs d'extinction des spermatozoïdes prélevés dès l'éjaculation ou après 18 et $20 \mathrm{~h}$ de séjour dans l'utérus de brebis sont présentées dans le tableau 1. Sur 10 éjaculats, la teneur moyenne en ADN-UV des spermatozoïdes ne semble pas affectée par leur présence prolongée dans les voies génitales femelles, à l'exception de deux cas où une faible augmentation est observée. Par contre, leur contenu en ADN-Feulgen augmente de manière notable pour 8 des 10 échantillons observés après 18 à 20 h de séjour in utero.

b) Evolution de I' "ADN-Feulgen » selon le temps de présence in utero. - Chez 59 des 98 brebis, réparties dans tous les lots de l'expérience, les spermatozoïdes ont montré une augmentation significative de la colorabilité du matériel nucléaire par la réaction de Feulgen. Le tableau 2 rapporte la moyenne ( \pm erreur-standard) des taux individuels d'augmentation dans chacun des lots (temps de séjour).

La proportion des brebis chez qui les spermatozoïdes ont réagi semble progresser par paliers en fonction du temps de présence des gamètes dans les voies génitales 
TABLEAU 1

Evolution comparée de l'ADN, mesuré en UV ou après coloration de Feulgen, des spermatozoïdes de bélier, éjaculés ou après séjour in utero ${ }^{1}$ )

\begin{tabular}{|c|c|c|c|c|c|}
\hline \multicolumn{2}{|c|}{ Extinction UV } & \multirow{2}{*}{$\begin{array}{l}\text { Variation } \\
\text { de l'extinction } \\
\text { (p. 100) }\end{array}$} & \multicolumn{2}{|c|}{ Extinction Feulgen } & \multirow{2}{*}{$\begin{array}{l}\text { Variation } \\
\text { de l'extinctior } \\
\text { (p. 100) }\end{array}$} \\
\hline Spz éjac. & Spz ut. $\left({ }^{2}\right)$ & & Spz. éjac. & Spz ut. $\left({ }^{2}\right)$ & \\
\hline $\begin{array}{l}5,99 \pm 0,03 \\
5,75 \pm 0,03 \\
5,88 \pm 0,04 \\
6,01 \pm 0,03 \\
5,96 \pm 0,03 \\
5,95 \pm 0,03 \\
5,95 \pm 0,03 \\
5,95 \pm 0,03 \\
5,99 \pm 0,03 \\
5,97 \pm 0,03\end{array}$ & $\begin{array}{l}5,99 \pm 0,04 \\
5,92 \pm 0,03 \\
5,92 \pm 0,04 \\
6,04 \pm 0,02 \\
6,11 \pm 0,04 \\
6,02 \pm 0,03 \\
5,95 \pm 0,03 \\
5,99 \pm 0,04 \\
6,06 \pm 0,04 \\
5,97 \pm 0,03\end{array}$ & $\begin{array}{l}\overline{2,9} \\
- \\
\overline{2,5} \\
= \\
= \\
=\end{array}$ & $\begin{array}{l}4,85 \pm 0,05 \\
4,02 \pm 0,03 \\
3,58 \pm 0,05 \\
4,66 \pm 0,05 \\
3,31 \pm 0,13 \\
3,26 \pm 0,09 \\
4,36 \pm 0,05 \\
4,58 \pm 0,07 \\
4,78 \pm 0,07 \\
4,63 \pm 0,06\end{array}$ & $\begin{array}{l}4,85 \pm 0,08 \\
4,40 \pm 0,04 \\
3,82 \pm 0,03 \\
4,97 \pm 0,05 \\
4,69 \pm 0,15 \\
4,17 \pm 0,10 \\
4,79 \pm 0,06 \\
4,87 \pm 0,08 \\
4,78 \pm 0,07 \\
5,31 \pm 0,08\end{array}$ & $\begin{array}{r}\overline{9}, 4 \\
6,7 \\
6,7 \\
41,7 \\
27,9 \\
9,9 \\
6,3 \\
\overline{14,7}\end{array}$ \\
\hline
\end{tabular}

(1) Une ligne du tableau représente les résultats d'un éjaculat. Les résultats sont exprimés en unités arbitraires $(\bar{m} \pm$ sem).

$\left({ }^{2}\right)$ Le temps de séjour dans les voies génitales femelles est de $20 \mathrm{~h}$ pour les 4 premiers échantillons et $18 \mathrm{~h}$ pour les autres.

femelles : dès $2 \mathrm{~h}$, la moifié des échantillons présente une réaction, cette valeur reste à peu près la même jusqu'à $16 \mathrm{~h}$ puis augmente jusqu'à la totalité des échantillons $24 \mathrm{~h}$ après l'accouplement. L'influence du milieu utérin se traduit aussi par des modifications de la taille des noyaux des spermatozoïdes (tabl. 2). Celle-ci est assez variable

\section{TABLEAU 2}

Augmentation du matériel ADN-Feulgen et changement de taille nucléaire des spermatozoìdes de bélier selon le temps de séjour dans l'utérus des brebis

\begin{tabular}{|c|c|c|c|c|}
\hline $\begin{array}{c}\text { Temps de séjour } \\
\text { dans l'utérus } \\
\text { (h) }\end{array}$ & $\begin{array}{c}\text { Nbre de? } \\
\text { saillies }\end{array}$ & $\begin{array}{l}\text { Nbre de } \text { Q avec } \\
\text { "ADN-Feulgen 》 } \\
\text { augmenté }(1)\end{array}$ & $\begin{array}{l}\text { Augmentation } \\
\text { moyenne } \\
\left(\text { p. 100) }\left(^{2}\right)\right.\end{array}$ & $\begin{array}{l}\text { Changement moyen } \\
\text { de surface nucléaire } \\
\left(\text { p. 100) }{ }^{3}\right)\end{array}$ \\
\hline $\begin{array}{r}24 \\
22 \\
20 \\
18 \\
16 \\
14 \\
12 \\
10 \\
8 \\
6 \\
4 \\
2\end{array}$ & $\begin{array}{r}10 \\
8 \\
8 \\
8 \\
8 \\
8 \\
8 \\
8 \\
8 \\
8 \\
8 \\
8\end{array}$ & $\begin{array}{r}10 \\
7 \\
5 \\
7 \\
4 \\
6 \\
4 \\
1 \\
4 \\
3 \\
4 \\
4\end{array}$ & $\begin{array}{r}28,9 \pm 5,9 \\
20,8 \pm 5,6 \\
8,1 \pm 1,4 \\
17,4 \pm 4,8 \\
12,5 \pm 1,1 \\
18,1 \pm 4,2 \\
13,0 \pm 3,9 \\
4,9 \\
9,6 \pm 2.6 \\
7,8 \pm 3,6 \\
15,7 \pm 6,6 \\
13,7 \pm 6,1\end{array}$ & $\begin{array}{r}12,5 \pm 4,4 \\
8,1 \pm 1,8 \\
2,3 \pm 1,2 \\
4,6 \pm 1,2 \\
0,9 \pm 1,1 \\
1,9 \pm 2,5 \\
0,2 \pm 0,7 \\
0,3 \\
5,1 \pm 5,0 \\
-1,9 \pm 1,0 \\
4,6 \pm 2,3 \\
-\end{array}$ \\
\hline
\end{tabular}

( $\left.{ }^{1}\right)$ Femelles chez qui le contenu en ADN-Feulgen des spermatozoïdes est significativement supérieur à celui des spermatozoīdes éjaculés.

(2) Moyenne des taux individuels d'augmentation ( $\overline{\mathrm{m}} \pm \mathrm{sem})$.

( $\left.{ }^{3}\right)$ Moyenne des taux individuels de modifications de surface des têtes de spermatozoïdes réactivés $(\mathrm{m} \pm \mathrm{sem})$. 
au début, mais une nette augmentation de surface est notée après 18 à 24 h de séjour dans le tractus femelle.

Par ailleurs, chez les 39 brebis où les spermatozoïdes sont considérés comme n'ayant pas réagi au milieu utérin, le contenu en ADN-Feulgen varie de la manière suivante : il augmente non significativement dans 14 cas, ne varie pas dans 8 et diminue dans les 17 autres cas dont 10 de manière significative. Il ne semble pas que le taux de diminution puisse être relié au temps de séjour utérin.

c) Protéines basiques. - La coloration au Fast-Green alcalin n'a été effectuée que sur 21 échantillons de spermatozoïdes (animaux traités au cours de la même saison).

Le séjour des spermatozoïdes dans les voies génitales femelles entraîne une augmentation de leur coloration par le Fast-Green alcalin (tabl. 3). Celle-ci est aléatoire

\section{TABLEAU 3}

Contenu en proféines basiques après coloration au Fast-Green alcalin des spermatozoïdes de bélier, au moment de l'éjaculation ou oprès séjour dans l'utérus de la brebis

\begin{tabular}{|c|c|c|c|c|}
\hline \multirow{2}{*}{$\begin{array}{l}\text { Temps } \\
\text { de séjour } \\
\text { utérin }\end{array}$} & \multicolumn{2}{|c|}{$\begin{array}{c}\text { Extinction Fast-Green } \\
*\end{array}$} & \multirow{2}{*}{$\begin{array}{l}\text { Variation de } \\
\text { l'extinction } \\
\text { (p. 100) }\end{array}$} & \multirow{2}{*}{$\begin{array}{c}\text { Spz utérins porteurs } \\
\text { de zones claires } \\
\text { (p. 100) }\end{array}$} \\
\hline & Spz éjaculés & Spz utérins & & \\
\hline 22 h.......... & $\begin{array}{r}8,96 \pm 0,15 \\
10,15 \pm 0,11 \\
9,6 \pm 0,09 \\
6,73 \pm 0,15\end{array}$ & $\begin{array}{r}12,28 \pm 0,19 \\
11,19 \pm 0,13 \\
11,80 \pm 0,15 \\
8,34 \pm 0,16\end{array}$ & $\begin{array}{l}37,0 \\
10,2 \\
22,9 \\
23,9\end{array}$ & $\begin{array}{l}40,7 \\
11,4 \\
\overline{67,0}\end{array}$ \\
\hline $16 \mathrm{~h} \ldots \ldots \ldots \ldots$ & $5,37 \pm 0,09$ & $6,28 \pm 0,08$ & 16,9 & - \\
\hline 14 h.......... & $\begin{array}{l}6,40 \pm 0,12 \\
7,41 \pm 0,10\end{array}$ & $\begin{array}{r}10,66 \pm 0,33 \\
8,08 \pm 0,07\end{array}$ & $\begin{array}{r}66,6 \\
9,0\end{array}$ & $\overline{31,0}$ \\
\hline 12 h.......... & $\begin{array}{l}6,74 \pm 0,10 \\
7,42 \pm 0,11\end{array}$ & $\begin{array}{l}8,02 \pm 0,10 \\
8,25 \pm 0,08\end{array}$ & $\begin{array}{l}19,0 \\
11,2\end{array}$ & $\overline{15,5}$ \\
\hline $10 \mathrm{~h} . \ldots \ldots \ldots$. & $\begin{array}{l}7,03 \pm 0,10 \\
6,94 \pm 0,09\end{array}$ & $\begin{array}{l}8,70 \pm 0,10 \\
7,09 \pm 0,11\end{array}$ & $\begin{array}{r}23,8 \\
2,2\end{array}$ & - \\
\hline $8 \mathrm{~h} \ldots \ldots \ldots \ldots$ & $\begin{array}{l}6,84 \pm 0,20 \\
7,14 \pm 0,11\end{array}$ & $\begin{array}{r}10,66 \pm 0,17 \\
9,01 \pm 0,32\end{array}$ & $\begin{array}{l}55,8 \\
26,2\end{array}$ & - \\
\hline $6 \mathrm{~h} \ldots \ldots \ldots \ldots$ & $\begin{array}{l}9,31 \pm 0,12 \\
7,84 \pm 0,09 \\
9,40 \pm 0,07\end{array}$ & $\begin{array}{l}9,49 \pm 0,08 \\
9,52 \pm 0,07 \\
9,64 \pm 0,07\end{array}$ & $\begin{array}{r}1,9 \\
21,4 \\
2,6\end{array}$ & $\frac{18,6}{-}$ \\
\hline $4 h, \ldots \ldots \ldots$ & $\begin{array}{r}12,30 \pm 0,22 \\
6,93 \pm 0,19 \\
9,36 \pm 0,10\end{array}$ & $\begin{array}{r}11,69 \pm 0,26 \\
8,94 \pm 0,12 \\
9,71 \pm 0,12\end{array}$ & $\begin{array}{r}5,0 \\
29,0 \\
3,7\end{array}$ & $\begin{array}{l}\overline{41,9} \\
30,0\end{array}$ \\
\hline $2 h \ldots \ldots \ldots \ldots$ & $\begin{array}{l}10,33 \pm 0,34 \\
10,04 \pm 0,10\end{array}$ & $\begin{array}{r}10,88 \pm 0,17 \\
9,67 \pm 0,13\end{array}$ & $\begin{array}{r}5,3 \\
-3,7\end{array}$ & $\begin{array}{l}17,0 \\
34,2\end{array}$ \\
\hline
\end{tabular}
traires.

* Moyenne d'extinction ( \pm erreur standard) de 30 spermatozoïdes, exprimée en unités arbi- 
au début ( 2 et $4 \mathrm{~h}$ ). Très variable par la suite, elle concerne cependant tous les échantillons; elle tend à augmenter et à devenir un peu plus homogène à partir de $12 \mathrm{~h}$.

D'autre part, l'observation au microscope, montre des différences dans la distribution du matériel nucléaire coloré : chez les spermatozoïdes témoins, la coloration est intense dans la partie antérieure el postérieure, elle est faible juste après la zone équatoriale (fig. 1). Dans les spermatozoïdes « utérins », la coloration esł plus impor-

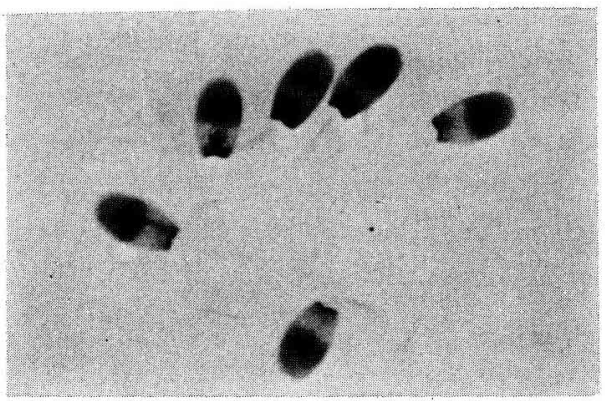

FIG. 1. - Spermatozoïdes éjaculés-témoins.

Coloration Fast-Green alcalin. $\mathrm{G} \times 1000$.

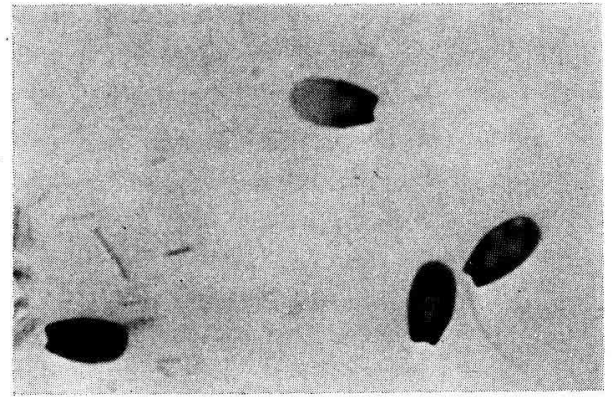

FIG. 2. - Spermatoïdes incubés in utero. Coloration Fast-Green alcalin. Exemple de noyaux porteurs d'altérations contrastant avec un spermatozoïde normal. $\mathrm{G} \times 1000$.

tanfe et sa répartition différente de celle des témoins : la zone équatoriale est moins marquée, parfois même non décelable. En outre, sur 10 des 21 échantillons, on note la présence d'une zone claire sous la forme d'une plage située longitudinalement dans la région post-acrosomique ef qui s'étend parfois dans la région recouverte par l'acrosome (fig. 2).

d) Groupements disulfures. - Les résultats, présentés au tableau 4, portent sur un échantillonnage choisi afin de mettre en évidence une éventuelle modification de

TABLEAU 4

Evolution comparée des groupements disulfures (coloration de Barrnett et Seligman) et du matériel ADN-Feulgen

Variations de l'extinction du matériel coloré (p. 100)

\begin{tabular}{cccc}
\hline Feulgen augmenté & Groupes S-S & Feulgen diminué & Groupes S-S \\
\hline$+2,7$ & $-3,6$ & $-4,4$ & $-5,4$ \\
$+29,1$ & $-22,5$ & $-1,9$ & $+8,0$ \\
$+6,1$ & $+11,0$ & $-20,6$ & $+5,5$ \\
$+13,5$ & $\pm 2,9$ & $-6,2$ & $+11,0$ \\
$+4,1$ & $-14,8$ & $-6,8$ & $+3,0$
\end{tabular}

* La dernière ligne du tableau représente le taux moyen de variation du matériel coloré. 
la quantité de liaisons disulfures en relation avec le niveau du matériel ADN-Feulgen. Dans ce but, nous avons retenu 5 éjaculats dont les spermatozoïdes montraient une augmentation de la coloration de Feulgen ( +11 p. 100 en moyenne) et 5 éjaculats pour lesquels le taux de coloration était diminué ( -7 p. 100 en moyenne).

L'analyse statistique montre qu'il n'y a aucune corrélation entre les modifications de l'ADN-Feulgen et celles du nombre de liaisons disulfures. Toutefois, les mesures microspectrophotométriques montrent en moyenne une diminution de la coloration des ponts disulfures des spermatozoïdes du premier lot $(-6,5$ p. 100) et une augmentation dans l'autre lot ( +3 p. 100).

\section{Discussion.}

Tout ce travail a été fait avec des éjaculats de qualité normale, autant que l'on puisse en juger par la motilité des spermatozoïdes.

Les résultats des mesures cytophotométriques en lumière UV révèlent la stabilité de la teneur en ADN des noyaux de spermatozoïdes au long du séjour utérin. Cette stabilité fait suite à celle qui a déjà été observée lors de la spermiogenèse et de la maturation épididymaire (Esnault ef Nicolle, 1976).

Au contraire, la teneur moyenne des spermatozoïdes en ADN-Feulgen, augmente dans 60 p. 100 des échantillons qui ont séjourné in utero, surtout après les plus longs délais. Un tel phénomène avait déjà été remarqué avec les spermatozoïdes de lapins (Esnault, Orgebin-Crist, Ortavant, cités par Orgebin-Crist, 1969).

Parailèlement à cette réaction, nous observons une augmentation de la surface des spermatozoïdes, elle aussi plus importante après les temps de séjour longs ; ceci reflète une certaine progression dans la décondensation de la chromatine, qui s'opère dans les voies génitales de la femelle (tabl. 2).

Il semble donc que les modifications quantitatives du matériel coloré reflètent l'évolution de la structure de la chromatine ; dans le cas présent, il s'agit d'un démasquage accru de l'ADN, plus accessible à la leucofuschine. Un processus inverse à celui observé ici a été rapporté pendant la spermiogenèse et la maturation épididymaire des gamètes, à savoir, une diminution de la colorabilité Feulgen du matériel r.Jcléaire, associée à la condensation de la chromatine (Esnault, 1965 ; Gledhill ef al., 1966), chez le taureau (Esnault et Nicolle, 1976) chez le bélier.

La coloration de Barrnett et Seligman ne confirme pas que l'augmentation de colorabilité de l'ADN par la réaction de Feulgen obtenue in utero est liée à une diminution des liaisons disulfures comme le laissaient supposer les résultats obtenus sur des spermatozoïdes éjaculés traités par le dithiothreitol (Esnault, 1973). Les résultats qu'e nous observons ici sont peut-être dus à la technique de Barrnett et Seligman ellemême ; ils doivent être considérés avec réserve car les niveaux d'extinction sont faibles $(1,02$ à 1,62), à la limite de la sensibilité photométrique et la stœchiométrie de la réaction n'est pas vérifiée, la réduction chimique au thioglycérol des groupements disulfures (Gomori, 1956) n'étant elle-même pas contrôlable quantitativement. D'autre part, avec un matériel aussi condensé que la chromatine des spermatozoïdes, il est vraisemblable que des réducteurs tels que le dithiothreitol et le thioglycérol ne sont pas assez efficaces pour atteindre la totalité des groupements disulfures. La réduc- 
tion complète de ces liaisons nécessite de travailler en milieu dénaturant (chlorure de guanidine et urée). Un tel traitement conduit à la dispersion du noyau qui ne peut être, de ce fait, suivi par des mesures cytophotométriques.

Par autoradiographie avec de l'arginine marquée, Kopecny ef Fulka (1975) ont montré la stabilité de la protéine spécifique du spermatozoïde de lapin jusqu'à l'entrée de celui-ci dans l'ovocyle ; ceci traduit une stabilité du rapport en quantité de l'ADN ef des protéines basiques. L'augmentation de la coloration au Fast-Green alcalin des spermatozoïdes « utérins » que nous observons semble donc plutôt liée à la qualité ou à la configuration de la chromatine. Cette méthode de coloration des protéines basiques est très élective (Lison, 1960). Nos résultats suggèrent que l'augmentation de colorabilité que nous observons pour les spermatozoïdes « utérins 》 est facilitée par une liaison moins étroite des molécules protéiques entre elles, consécutive à une modification de leur structure. Noeske (1973) suggère que l'augmentation de coloration au Fast-Green, qu'il met en évidence dans les noyaux pendant la maturation des cellules myélopoiétiques, serait due à un blocage moindre des groupements aminés des histones.

La présence de zones claires dans certains spermatozoïdes, rappelle les vacuoles que mettent en évidence, Motta et Van Blerkom (1975), chez le lapin, Kann et Fouquet (1977), chez le taureau, à la surface de spermatozoïdes incubés in utero et qu'ils interprètent comme étant le résultat de la rupture de la membrane plasmique post-acrosomique. On peut se demander si les modifications de la chromatine sont la conséquence des déséquilibres observés au niveau des membranes plasmiques et acrosomiques (régions du spermatozoïde en contact direct avec les agents chimiques, enzymatiques et hormonaux du milieu utérin). Ne seraient-elles pas la manifestation d'une phase préparant ainsi la chromatine à son gonflement dans le cytoplasme de l'œuf lors de la fécondation ? Ou bien ces vacuoles étaient déjà présentes à la surface de certains spermatozoïdes éjaculés (témoins) mais à un état moins avancé et non décelable par notre technique (Hrudka et Zibrin, 1964 ; Saacke et Almquist, 1964 ; Bane et Nicander, 1965 ; Morstin et Courot, 1974 ; Coulter, Oko et Costerton, 1978). La présence de telles anomalies serait liée à une moins bonne fécondance (Morstin et Courot, 1974 ; Uzu, Courtens, Courot, 1976) ef les changements que nous observons après séjour des spermatozoïdes dans les voies génitales femelles pourraient alors refléter un état de dégénérescence de tels spermatozoïdes. Dans ce cas, il serait possible de supposer que les spermatozoïdes n'ayant montré aucune modification morphologique pendant le transit utérin seraient les seuls capables d'assurer la fécondation.

En conclusion, il apparaît que le stock d'ADN acquis par le gamète mâle lors de sa formation reste inchangé jusqu'à la fécondation. Les nucléoprotéines ont un rôle de support et de protecteur vis-à-vis de l'ADN. Les transformations structurales qu'elles subissent pendant le transit utérin semblent constifuer une phase nécessaire et préalable à la fusion du spermatozoïde avec l'œuf. Ces modifications sont induites par le milieu utérin qui joue ainsi un rôle de sélection, par l'élimination d'une proportion importante de spermatozoïdes dont on peut penser qu'ils n'auraient pas été aptes à assurer la fécondation de manière satisfaisante. 


\section{Références}

ALFERT M., GESCHWIND I. I., 1953. A selective staining method for the basic proteins of cell nuclei. Proc. nat. Acad. Sci., 39, 991-999.

BAHR G. F., MOBERGER G., 1958. Histochemical method for the demonstration of sulfhydryl groups in normal tissues and malignant fumors. Acta pathol. microbiol. scand., 42, 109-132.

BANE A., NICANDER L., 1965. Pouch formations by invaginations of the nuclear envelope of bovine and porcine sperm as a sign of disturbed spermiogenesis. Nord. vet. Med., 17, 628-632.

BARRNETT R. J., SELIGMAN A. M., 1952. Hisfochemical demonstration of protein bound sulfhydryl groups. Science, 116, 323-327.

CASPERSSON T., 1936. Über den chemischen Aufbau der Struckfuren des Zellkernes. Skand. Arch. Physiol., 73, suppl. 8.

COULTER G. H., OKO R. J., COSTERTON J. W., 1978. Incidence and ultrastructure of " crater " defect of bovine spermatozoa. Theriogenology, 9, 165-173.

ESNAULT C., 1965 Cité par COUROT M., HOCHEREAU-de-REVIERS M. T., ORTAVANT R., Spermatogenesis. In JOHNSON A. D., GOMES W. R., VANDEMARK N. L. The testis. Acod. Press Inc. N. Y., 1, 339-432.

ESNAULT C., 1973. Reactivation of the Feulgen reaction of ram spermatozoa by dithiothreitol. J. Reprod. Fert., 32, 153-157.

ESNAULT C., NICOLLEJ. C., 1976. Evolution de l'ADN et des protéines nucléaires basiques au cours de la maturation des cellules germinales du bélier. Etude microspectrophotométrique. Ann. Histochim., 21, 189-197.

GLEDHILL B. L., GLEDHILL M. P., RIGLER R. Jr., RINGERTZ N. R., 1966. Changes in deoxyribonucleoprotein during spermiogenesis in the bull. Exp. Cell Res., 41, 652-665.

GOMORI G., 1956. Histochemical methods for protein bound sulphydryl and disulphide groups. Quart. J. micr. Sci., 97, 1-9.

HRUDKA F., ZIBRIN M., 1964. The incidence of vacuoles in the nucleoplasma of normal bull spermia. Vet. Med. (Prague), 37, 11-18.

KANN M. L., FOUQUET J. P., 1977. Bull spermatozoa in the female tract after natural mating. A preliminary ultrastructural study of uterotubal junction. Ann. Biol. anim. Bioch. Biophys., 17, $165-172$.

KOPECNY V., FULKA J., 1975. Retention of nuclear basic protein in rabbit spermatozoa up to entry into the vitellus. Ann. Biol. anim. Bioch. Biophys., 15, 119-122.

LISON L., 1960. Histochimie et cytochimie animales. Gauthier-Villars Ed., Paris, 1, 343-344.

LOIR M., 1972a. Métabolisme de l'acide ribonucléique et des protéines dans les spermatocytes et les spermatides du bélier (Ovis aries) II. Variation de l'incorporation et devenir de la ${ }^{3} \mathrm{H}$ lysine, de la ${ }^{3} \mathrm{H}$ arginine et de la ${ }^{35} \mathrm{~S}$-cystine. Ann. Biol. anim. Bioch. Biophys., 12, 411-429.

LOIR M., 1972b. Métabolisme de l'acide ribonucléique et des protéines dans les spermatocytes et les spermatides du bélier (Ovis aries). III. Ełude cytochimique des transformations protéiques dans les vieilles spermatides. Ann. Biol. anim. Bioch. Biophys., 12, 531-544.

MARUSHIGE Y., MARUSHIGE K., 1975. Transformation of sperm histone during formation and maturation of rat spermatozoa. J. biol. Chem., 250, 39-45.

MONFOORT C. H., SCHIPHOF R., ROZIJN T. H., STEIN-PARVE E. P., 1973. Amino acid composition and carboxyl-terminal structure of some basic chromosomal proteins of mammalian spermatozoa. Biochim. biophys. Acta, 322, 173-177.

MORSTIN J., COUROT M., 1974. Ultrastructure des spermatozoïdes de taureaux de différente fécondance. Morphologie ultrastructurale, glycoprotéines acrosomiques et membranaires, charges négatives de surface. Ann. Biol. onim. Bioch. Biophys., 14, 581-590.

MOTTA P., VAN BLERKOM J., 1975. A scanning electron microscopic study of rabbit spermatozoa in the female reproductive tract following coïtus. Cell Tiss. Res., 163, 29-44.

NOESKE K., 1973. Discrepancies between cytophotometric alkaline Fast-Green measurements and nuclear histone protein content. Histochem. J., 5, 303-311.

ORGEBIN-CRIST M. C., 1969. Studies on the function of the epididymis. Biol. Reprod., Suppl. 1, 155-175.

SAACKE R. G., ALMQUIST J. O., 1964. Ultrastructure of bovine spermatozoa. The head of normal, ejaculated sperm. Amer. J. Anat., 115, 143-162.

UZU G., COURTENS J. L., COUROT M., 1976. Quantitative analysis of ultrastructural abnormalities of spermatozoa from bulls of different fertility. VIIIth int. Congr. Anim. Reprod. A. I., Krakow, 4, 748-751. 